\title{
Complex Oscillatory Yielding of Model Hard-Sphere Glasses
}

\author{
N. Koumakis, ${ }^{1}$ J. F. Brady, ${ }^{2}$ and G. Petekidis ${ }^{1, *}$ \\ ${ }^{1}$ FORTH/IESL and Department of Materials Science and Technology, University of Crete, 71110 Heraklion, Greece \\ ${ }^{2}$ Division of Chemistry and Chemical Engineering, California Institute of Technology, Pasadena, California 91125, USA
} (Received 1 February 2013; published 23 April 2013)

\begin{abstract}
The yielding behavior of hard sphere glasses under large-amplitude oscillatory shear has been studied by probing the interplay of Brownian motion and shear-induced diffusion at varying oscillation frequencies. Stress, structure and dynamics are followed by experimental rheology and Browian dynamics simulations. Brownian-motion-assisted cage escape dominates at low frequencies while escape through shear-induced collisions at high ones, both related with a yielding peak in $G^{\prime \prime}$. At intermediate frequencies a novel, for hard sphere glasses, double peak in $G^{\prime \prime}$ is revealed reflecting both mechanisms. At high frequencies and strain amplitudes a persistent structural anisotropy causes a stress drop within the cycle after strain reversal, while higher stress harmonics are minimized at certain strain amplitudes indicating an apparent harmonic response.
\end{abstract}

DOI: 10.1103/PhysRevLett.110.178301

PACS numbers: $83.80 . \mathrm{Hj}, 83.10 . \mathrm{Mj}$

Hard sphere (HS) colloids have been used as model systems to study a plethora of fundamental condensed matter problems such as the interplay between equilibrium phases (crystals and liquids) and nonergodic states (glasses or gels), and their behavior under external fields such as shear $[1,2]$. A major goal is to develop an understanding that will enable tailoring of the mechanical and flow properties based on the structure and dynamics at the particle level. HSs form metastable glasses above a volume fraction of about $\varphi_{g} \sim 0.59$ where crystallization and long-time diffusion are suppressed $[3,4]$. Such states exhibit solidlike behavior at low stresses or strains and shear-melting (yielding) above a yield stress or strain [5]. This phenomenon is investigated more effectively with a combination of rheological and optical or scattering techniques that may unravel the link between microstructure, dynamics, and mechanical properties in steady [6,7] or oscillatory shear $[8,9]$. Such studies have shown that particle cages are deformed under shear and particles move irreversibly when a critical strain is exceeded, leading to flow.

Experimentally, large-amplitude oscillatory shear tests are widely used to monitor yielding due to their simplicity and relation with linear elastic and viscous moduli, $G^{\prime}$ and $G^{\prime \prime}$. However, their interpretation in the nonlinear regime becomes complex due to stress distortion introducing higher harmonics [10]. In a wide range of systems (emulsions, polymers, and colloids) a generic peak in $G_{1}^{\prime \prime}$ (viscous modulus of the fundamental frequency) is observed representing an increased energy dissipation near the yield strain, $\gamma_{y}$, where $G_{1}^{\prime}=G_{1}^{\prime \prime}$, beyond which the sample flows [9,11]. On the theory side, mode coupling theory (MCT) [12] as well as the semi-phenomenological soft glassy rheology [13] are able to capture some aspects of largeamplitude oscillatory shear (LAOS) such as the peak of $G_{1}^{\prime \prime}$. Nevertheless, the underlying mechanisms relating stress with shear induced structure such as cage deformation, breaking, and reformation, as well as particle displacements, are poorly understood, and the frequency dependence is largely unexplored.

LAOS experiments are expected to provide valuable information on the interplay of Brownian motion and shear during yielding of HS glasses. Along this line, light scattering-echo experiments probing the average particle displacements under oscillatory shear $[8,9]$ revealed a transition to irreversibility beyond a critical strain amplitude $[8,9]$. This can be viewed as the analogue of the shear induced irreversibility observed in concentrated nonBrownian particles [14], since, at rest, diffusion is absent at all length scales in non-Brownian particles, while in colloidal glasses only the out-of-cage diffusion is frozen. While experiments combining rheometry with scattering or microscopy are rather demanding [8,15-18], computer simulations can provide an alternative route.

Here, we use a combination of oscillatory shear rheometry and Brownian dynamics (BD) simulations to investigate the links between structure and particle dynamics with the nonlinear rheological response of HS glasses in a wide range of frequencies, $\omega$, nondimensionalized by Peclet number $\mathrm{Pe}_{\omega}^{0}=\omega \tau_{B}$, with $\tau_{B}=R^{2} / D_{0}, R$ the radius and $D_{0}$ the free diffusion coefficient, or $\mathrm{Pe}_{\omega}$ if the short-time self diffusion coefficient $D_{s}(\varphi)$ is used. At low $\mathrm{Pe}_{\omega}^{0}$ yielding is related to Brownian-assisted irreversible particle motion manifested in a dynamic strain sweep (DSS) with the peak of $G_{1}^{\prime \prime}$. However, at the largely unexplored regime of high $\mathrm{Pe}_{\omega}^{0}$, we detect collision-dominated yielding and, within the oscillation period, a strongly anisotropic structure causing a reduced stress beyond strain reversal (memory of structure due to lack of Brownian relaxation). At intermediate $\mathrm{Pe}_{\omega}^{0}$, the sample is affected by both mechanisms as manifested by a novel, for HS glasses, double peak in $G_{1}^{\prime \prime}$.

We used model sterically stabilized poly(methyl methacrylate) nearly hard-sphere particles with two radii, 
$R=358$ and $130 \mathrm{~nm}$ (polydispersity 10\%-12\%) suspended in an octadecene and octadecene-bromonapthalene mixture respectively, in order to expand the $\mathrm{Pe}_{\omega}$ range. We prepared different volume fractions, $\varphi$, by diluting a random close packed batch with the exact $\varphi$, then adjusted by matching $G_{1}^{\prime}$ of the two systems in agreement with Ref. [19]. Experiments were performed on an ARES strain controlled rheometer (with $25 \mathrm{~mm}$ diameter, $0.01 \mathrm{rad}$ angle cone plate) and a solvent trap to eliminate evaporation. In BD simulations (described in the Supplemental Material [20]), HS interactions were implemented through the potential free algorithm [21]. Oscillatory shear was applied with periodic boundary conditions using typically 5405 particles with $10 \%$ polydispersity to avoid crystallization.

In Fig. 1, we show DSS tests performed at low and high $\mathrm{Pe}_{\omega}$, together with the evolution of the normalized intensity of all higher stress harmonics, $I_{\text {all }} / I_{1}=\sum I_{i} / I_{1}$, $(i=2 n+1, \quad n \geq 1)$, as well as Lissajous curves (intracycle stress versus strain) for representative strain amplitudes, $\gamma_{0}$. The low $\mathrm{Pe}_{\omega}(=0.5)$ data [Fig. 1(a)] exhibit the typical DSS response with a $G_{1}^{\prime \prime}$ peak around $\gamma_{y}\left(\right.$ at $G_{1}^{\prime}=G_{1}^{\prime \prime}$ ) similar to previous studies [11,19,22]. The nonlinear response is accompanied by progressively larger intracycle nonlinearities as indicated both by the Lissajous curves and the increasing $I_{\text {all }} / I_{1}$ as expected $[10,19]$. The latter increases beyond yielding and reaches almost 30\% at high $\gamma_{0}$ as found previously [19]. Moreover, the Lissajous plots show a transition from a linear viscoelastic behavior (elliptical shape) at low $\gamma_{0}$, to a parallelogram pattern indicative of an intracycle sequence of elastic-plastic response at $\gamma_{0}>\gamma_{y}[10,19]$.
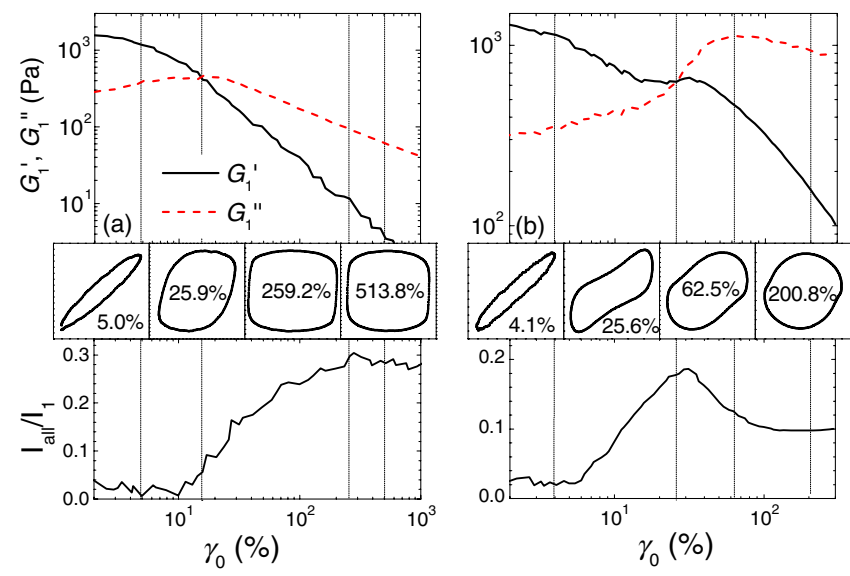

FIG. 1 (color online). Top: Dynamic strain sweeps for (a) $R=130 \mathrm{~nm}, \varphi=0.60$ at $\omega=1 \mathrm{rad} / \mathrm{s} \quad\left(\mathrm{Pe}_{\omega}^{0}=0.04\right.$, $\mathrm{Pe}_{\omega}=0.5$ ) and (b) $R=358 \mathrm{~nm}, \varphi=0.60$ at $\omega=1 \mathrm{rad} / \mathrm{s}$ $\left(\mathrm{Pe}_{\omega}^{0}=0.9, \mathrm{Pe}_{\omega}=11.2\right)$, with the 1 st harmonic of the elastic $G_{1}^{\prime}$ and viscous $G_{1}^{\prime \prime}$ modulus as a function of strain amplitude. Middle: Representative Lissajous plots are shown in different strain amplitudes as indicated. Bottom: Normalized total intensity of the higher harmonics of the stress, $I_{\text {all }} / I_{1}$.
At high $\mathrm{Pe}_{\omega}$, achieved with large particles $(R=358 \mathrm{~nm})$ at $\omega=1 \mathrm{rad} / \mathrm{s}\left(\mathrm{Pe}_{\omega}=11.2\right)$, the response is qualitatively different [Fig. 1(b)]. First, the peak of $G_{1}^{\prime \prime}$ shifts to higher $\gamma_{0}$, beyond $\gamma_{y}$. Second, $I_{\text {all }} / I_{1}$ (and individual $I_{2 n+1} / I_{1}$ ) exhibits a nonmonotonic behavior showing a first maximum around $\gamma_{y}$ and subsequently decreases substantially well inside the nonlinear regime. Hence, the sample exhibits a more harmonic stress response (anharmonicity is lowered) even though under nonlinear LAOS.

We further explore the $\mathrm{Pe}_{\omega}$ dependence by changing $\omega$ while keeping $\gamma_{0}$ constant. In Fig. 2(a), experiments with varying $\omega$ (at $\gamma_{0}=100 \%$ ) reveal the transition from the low-Pe ${ }_{\omega}$ rectangular shaped Lissajous curves reflecting a sequence of elastic and plastic responses, to the high-Pe ${ }_{\omega}$ regime with a characteristic ellipsoid with a double concave distortion caused by reduced stress in the quadrants II and IV after strain reversal. The intensity of the 3rd harmonic at $100 \%$ (above $\gamma_{y}$ ) exhibits a minimum with $\omega$ in experiments, $\varphi=0.62$, and $\mathrm{BD}, \varphi=0.60$, [Fig. 2(c)]; note that the position of minimum is $\varphi$ and $\gamma_{0}$ dependent. While in both $\mathrm{Pe}_{\omega}$ regimes the stress response is highly anharmonic, with significant $I_{3} / I_{1}$, during the transition, the Lissajous curves [Fig. 2(a)] acquire an ellipsoid shape involving almost zero higher harmonic contributions. BD simulations showing identical rheological response [Fig. 2(b)] with experiments are able to provide valuable structural information revealing the underlying mechanism of such stress reduction. In Fig. 2(d), we plot the 2D projection of the pair correlation function (calculated in a
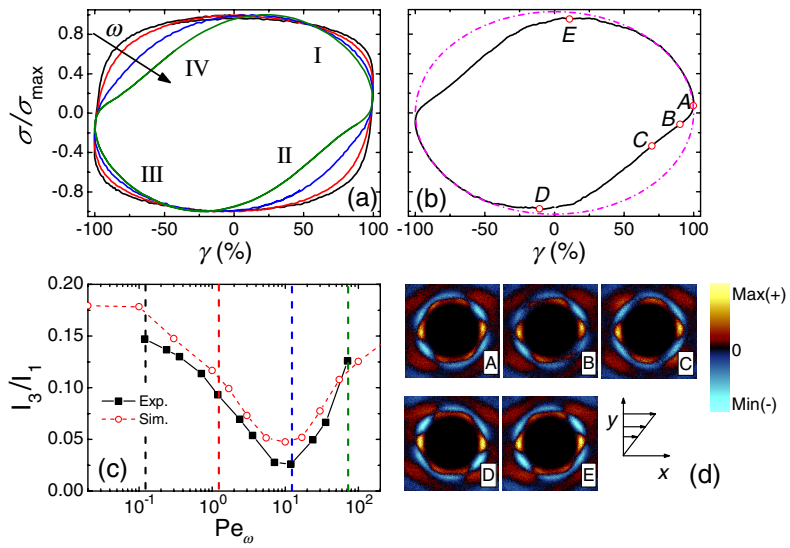

FIG. 2 (color online). Lissajous plots at $100 \%$ strain amplitude (a) for experiments at different $\mathrm{Pe}_{\omega}$ denoted by the dashed lines in (c) with the 4 quadrants of the oscillatory cycle indicated and (b) $\mathrm{BD}$ simulations at $\mathrm{Pe}_{\omega}=100$. The dashed line in (b) indicates a viscous harmonic stress strain response (see also Supplemental Material [20]). (c) $\mathrm{Pe}_{\omega}$ dependence of the $3 \mathrm{rd}$ harmonic at $100 \%$, for experiments at $\varphi=0.62(R=358 \mathrm{~nm})$ and BD simulations at $\varphi=0.60$. (d) $2 \mathrm{D}$ projections in the velocity-gradient $(x y)$ plane of the difference of $g_{x y}(r)$ under shear from that at rest from BD simulations at indicated points within the cycle, points $A-E$ in (b). 
thin slice of $\pm 0.7 R$ ) in the velocity-gradient $(x y)$ direction, $g_{x y}(r)$, at specific points inside the oscillation cycle for $\mathrm{Pe}_{\omega}=100$, similar to findings under steady shear [7]. Contrary to what we find at low $\mathrm{Pe}_{\omega}$ (Supplemental Material [20]), here the structure is highly anisotropic at the point of maximum strain [zero shear rate; point $A$ in Fig. 2(b)]. Moreover, such anisotropy is persistent during a large part of the successive quadrant where the shear has been reversed (points $B$ and $C$ ). Such an anisotropic cage, created during high $\mathrm{Pe}_{\omega}$ shear in one direction, allows flow with less stress (due to fewer particle collisions) when shear is reversed. Thus, in quadrants II and IV the stress is reduced if compared to a fully harmonic viscous response corresponding to the flowing anisotropic structure of quadrants I and III [dashed-dotted line in Fig. 2(b)]. Only beyond zero strain (points $D$ and $E$ ), is the structure reversed and the stress comes back to the maximum values within the period (see Supplemental Material [20]). Such a response is absent at low $\mathrm{Pe}_{\omega}$ since Brownian motion relaxes shear-induced structural anisotropy more efficiently, and the stress response is similar in all quadrants.

The data presented above verify the existence of the two $\mathrm{Pe}_{\omega}$ regimes: the low $\mathrm{Pe}_{\omega}$ one, conventionally studied up to now, where Brownian motion is dominant, and the high $\mathrm{Pe}_{\omega}$ where shear-induced particle collisions introduce novel LAOS features related to the persistent structural anisotropy and the consequent reduced stress after strain reversal. The transition from Brownian activated yielding, where particles under shear escape their cages assisted by thermal motion, to collision-induced cage breaking at high frequencies is linked to pronounced irreversible particle rearrangements and decreasing $\gamma_{y}$ at low frequencies $[8,9]$.

We further investigated HS glasses at higher $\varphi$ and frequencies corresponding to an intermediate ( $\varphi$ dependent) $\mathrm{Pe}_{\omega}$ regime. Experimental DSS, Lissajous plots, and $I_{\text {all }} / I_{1}$, shown in Fig. 3 as a function of $\gamma_{0}$ for $\varphi=0.639$, reveal an even richer mechanical response at $\mathrm{Pe}_{\omega}^{0}=0.4\left(\mathrm{Pe}_{\omega}=8\right)$. A main observation here is the unambiguous detection of two peaks in $G_{1}^{\prime \prime}$ detected for the first time in HS glasses. Such a feature was so far observed only in attractive glasses and gels indicating a two-step yielding due to two length scales present in attractive systems, i.e., the interparticle bond and the cage or cluster size [22,23]. However, the double $G_{1}^{\prime \prime}$ peak seen here must be of a different nature since a second length scale is absent, and moreover, the phenomenon is only observed in a narrow range of $\mathrm{Pe}_{\omega}$. The first peak of $G_{1}^{\prime \prime}$ is identified with the one observed at low $\mathrm{Pe}_{\omega}$ by a direct comparison of the two DSSs $\left(\mathrm{Pe}_{\omega}^{0}=0.04\right.$ and 0.4$)$ shown in Fig. 3(a). The two peaks signify the maximum in energy dissipation during the two yielding mechanisms at low and high $\mathrm{Pe}_{\omega}^{0}$, attributed to cage breaking via shearassisted activated hopping and through particle collisions, respectively. The Lissajous figures and higher harmonics

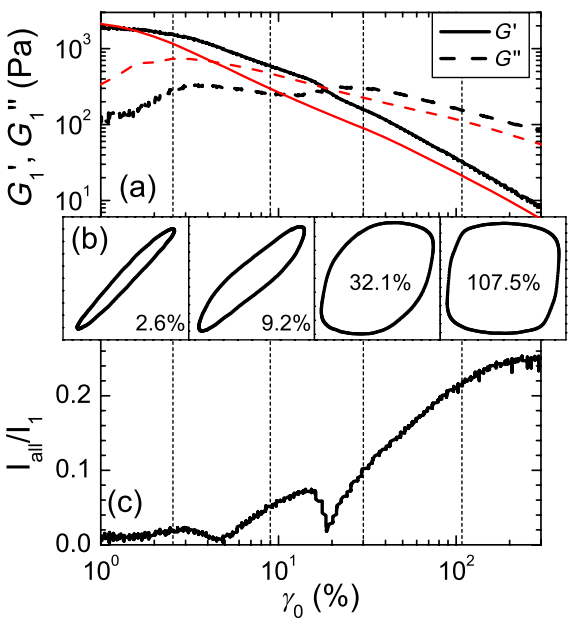

FIG. 3 (color online). (a) Dynamic strain sweeps with $R=130 \mathrm{~nm}$ particles at $\varphi=0.639$ and an intermediate $\left(\omega=10 \mathrm{rad} / \mathrm{s}, \mathrm{Pe}_{\omega}^{0}=0.4, \mathrm{Pe}_{\omega}=8\right.$ thick black lines $)$ and low $\left(\omega=1 \mathrm{rad} / \mathrm{s}, \mathrm{Pe}_{\omega}^{0}=0.04, \mathrm{Pe}_{\omega}=0.8\right.$ thin red lines $)$ frequency regime. (b) Indicative Lissajous plots for $\mathrm{Pe}_{\omega}=8$ at different strain amplitudes and (c) normalized total intensity of higher harmonics $I_{\text {all }} / I_{1}$, versus strain amplitude for $\mathrm{Pe}_{\omega}=8$.

reveal a transition from the low $\gamma_{0}$ linear response to a viscoplastic flow at high $\gamma_{0}$ passing through two states with an apparent harmonic response as indicated by the two minima of $I_{\text {all }} / I_{1}$. At these strain amplitudes the Lissajous curves acquire a nearly ellipsoidal shape due to the compensation of the structural phenomena during the transition from low to high $\mathrm{Pe}_{\omega}$ [as in Fig. 2(c)]. Note that for this sample $(R=130 \mathrm{~nm}, \varphi=0.639)$ the high $\mathrm{Pe}_{\omega}$ [Fig. 1(b)] was not within the experimental window.

Figure 4(a) shows $G_{1}^{\prime}$ and $G_{1}^{\prime \prime}$ from BD LAOS tests for $\varphi=0.60$ at different $\mathrm{Pe}_{\omega}$. The dependence of $G_{1}^{\prime}$ and $G_{1}^{\prime \prime}$ at high $\gamma_{0}\left(>\gamma_{y}\right)$ follows a power law decrease $G_{1}^{\prime}\left(G_{1}^{\prime \prime}\right) \propto$ $\gamma_{0}^{\nu^{\prime}}\left(\gamma_{0}^{v^{\prime \prime}}\right)$ as detected experimentally [11,19]. While Maxwell-type models give $\nu^{\prime}=2 \nu^{\prime \prime}=-2$ and MCT (around the glass transition) predicts lower values, but similar $\nu^{\prime} / \nu^{\prime \prime}$ ratio $[12,24]$ experiments in HS glasses show deviations from such simple dependency [19]. In agreement with experiments (Fig. 1), BD simulations give $\mathrm{Pe}_{\omega}$ dependent exponents [Fig. 4(a)] with $\nu^{\prime \prime}$ approaching -1 and 0 at low and high $\mathrm{Pe}_{\omega}$, respectively. Therefore, at high $\mathrm{Pe}_{\omega}$, where collision activated outof-cage particle rearrangements are dominant, $\gamma_{0} G_{1}^{\prime \prime} \simeq$ $\sigma\left(\dot{\gamma}_{\max }\right)$, a measure of energy dissipation per unit strain, is proportional to shear rate $\left(\gamma_{0} \omega\right)$ similar to the limiting high shear rate viscosity behavior under steady shear. On the other hand, at low $\mathrm{Pe}_{\omega}$, a $G_{1}^{\prime \prime} \propto \gamma_{0}^{-1}$ dependence corresponds to a steady shear thinning response of a HS glass at the yield stress plateau with $\eta_{\mathrm{eff}} \propto \dot{\gamma}^{-1}$.

We should note that the absence of hydrodynamic interactions (HI) in the BD simulations do not appear to introduce some qualitative difference as compared to experiments for the range of $\mathrm{Pe}_{\omega}$ studied here (up to about 

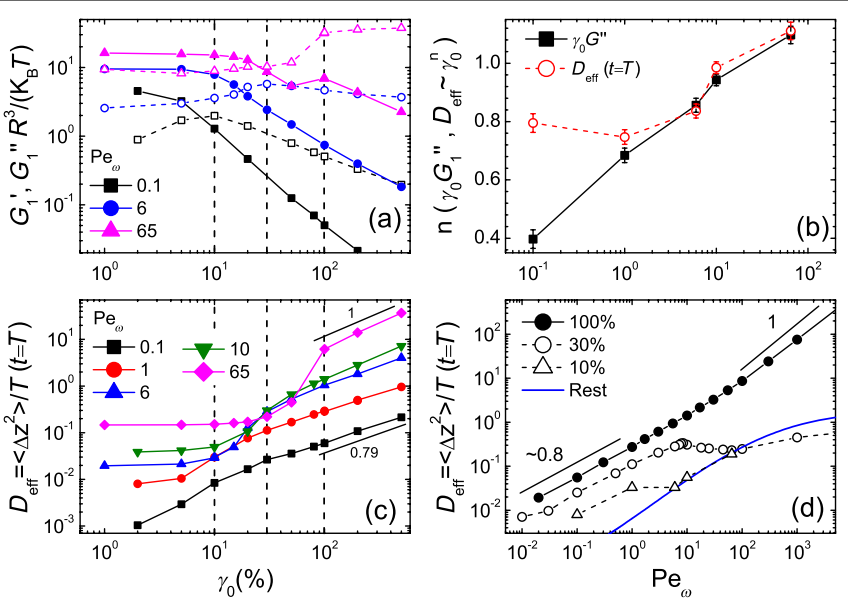

FIG. 4 (color online). BD simulations at $\varphi=0.60$ : (a) DSS data showing $G_{1}^{\prime}$ and $G_{1}^{\prime \prime}$ as a function of $\gamma_{0}$ for different $\mathrm{Pe}_{\omega}$ as indicated. (b) Power law exponent for the $\gamma_{0}$ dependence of $G_{1}^{\prime \prime}$ and $D_{\text {eff }}(t=T)$ as a function of $\mathrm{Pe}_{\omega}$. (c) Average $D_{\text {eff }}(t=T)$ versus $\gamma_{0}$ for different $\mathrm{Pe}_{\omega}$, and (d) $D_{\text {eff }}(t=T)$ versus $\mathrm{Pe}_{\omega}$ for $\gamma_{0}=10 \%, 30 \%$, and $100 \%$. The corresponding $D_{\text {eff }}(t=T)$ for a system at rest is also indicated.

100) as suggested by the similarity in the Lissajous plots (Fig. 2) and dynamic strain sweeps [Figs. 1 and 4(a)]. Along this line, the absence of a double $G_{1}^{\prime \prime}$ peak in BD cannot be attributed to the absence of hydrodynamic interactions, since BD at high volume fractions could not be conducted at a similar number of data points as experiments due to computational time restrictions. Nevertheless, a future direct comparison with Stokesian dynamics simulations (with full HI) would be quite valuable to pin-point details in the role of $\mathrm{HI}$ for $\mathrm{Pe}_{\omega}>1$.

Further insight into the two yielding mechanisms is gained by examining microscopic particle dynamics within a LAOS cycle by BD. In Fig. 4(c), we show the effective diffusivity, $D_{\text {eff }}(t=T)=\left\langle\Delta z^{2}(T)\right\rangle / T$ with $\left\langle\Delta z^{2}(T)\right\rangle$ the mean square displacement in the vorticity direction, $z$, (at $t=T$ ), as a function of $\gamma_{0}$ for several $\mathrm{Pe}_{\omega}$. At low $\mathrm{Pe}_{\omega}, D_{\text {eff }}(T)$ increases sublinearly with $\gamma_{0}$, whereas as $\mathrm{Pe}_{\omega}$ is increased it exhibits progressively a weaker increase at small $\gamma_{0}$ and a stronger one at higher $\gamma_{0}$. The constant $D_{\text {eff }}(T)$ for $\gamma_{0} \ll \gamma_{y}$ indicates that prior to yielding, incage diffusion is unaffected by shear; for the lowest $\mathrm{Pe}_{\omega}$, this regime is not reached since $\gamma_{y}$ increases with frequency [9]. For $\gamma_{0}>\gamma_{y}, D_{\text {eff }}(T)$, corresponding to out-of cage diffusion, increases sublinearly $\left[\gamma_{0}=100 \%\right.$, Fig. 4(d)] with a power law exponent $\simeq 0.8$ at low $\mathrm{Pe}_{\omega}$, which approaches 1 for $\mathrm{Pe}_{\omega}>1$ where direct particle collisions are dominant. In comparison, the corresponding $D_{\text {eff }}(T)$ for $\gamma_{0}=10 \%$ and $30 \%$ show an initial sublinear dependence at low $\mathrm{Pe}_{\omega}$ and subsequently approach the curve at rest, since for such frequencies $\gamma_{y}>30 \%$ [9] and the sample has not yet yielded. Note that a similar power law increase (with exponent $\sim 0.8$ ) has been detected in HS glasses at low steady shear rates by confocal microscopy [6], a weak but systematic deviation from the linear MCT prediction [25] and closer to agreement with nonlinear Langevin equation theory involving activated hopping mechanisms [26]. For our oscillatory BD, a linear dependence is reached for $\mathrm{Pe}_{\omega}>10$ as expected for nonBrownian particles under steady shear [27]. We suggest that the sublinear and linear dependencies probed here at low and high $\mathrm{Pe}_{\omega}$, respectively, reflect the two different mechanisms involved in the two regimes. Note that similarly with $D_{\text {eff }}, \gamma_{0} G_{1}^{\prime \prime}$ increases linearly with $\gamma_{0}$ at high $\mathrm{Pe}_{\omega}$; the linear $\gamma_{0}$ dependence of both quantities is linked to collision-induced yielding. On the other hand, at low $\mathrm{Pe}_{\omega}$ both quantities increase sublinearly, $D_{\text {eff }} \propto \gamma_{0}^{0.8}$ and $\gamma_{0} G_{1}^{\prime \prime} \propto \gamma_{0}^{0.4}$ (the latter exponent tends to zero as $\mathrm{Pe}_{\omega}$ is lowered) reflecting plastic flow, with a power law stress behavior, and complex Brownian motion and shearactivated particle hopping.

In summary, the combination of experimental oscillatory rheology and BD simulations has revealed the complete mechanical fingerprint of HS glasses and the related underlying microscopic structure and dynamics over a wide frequency regime where both Brownian and nonBrownian behavior is probed. At low $\mathrm{Pe}_{\omega}$, commonly studied up to now, Brownian-assisted irreversible motion takes place during yielding with a single peak of $G_{1}^{\prime \prime}$ and strong higher harmonics of the stress at large $\gamma_{0}$. In this regime, the microstructure under shear is only weakly anisotropic while the shear-induced diffusivity scales sublinearly with $\mathrm{Pe}_{\omega}$. At high $\mathrm{Pe}_{\omega}$, yielding is dictated by collision-induced displacements linked with a shearinduced long time diffusion that increases linearly with $\mathrm{Pe}_{\omega}$. A single $G_{1}^{\prime \prime}$ peak is detected at $\gamma_{0}>\gamma_{y}$ which eventually turns into a plateau at the limit of large $\mathrm{Pe}_{\omega}$. The structure under shear is strongly anisotropic, but more interestingly, exhibits a hysteresis under strain reversal. Such structural memory, due to lack of Brownian relaxation, causes a characteristic stress drop after strain reversal as revealed by the Lissajous curves. At some characteristic $\gamma_{0}$, higher harmonics drop to almost zero indicating an unexpected harmonic response even though the sample is under nonlinear shear. Finally, at a $\varphi$ dependent intermediate $\mathrm{Pe}_{\omega}$ regime, the sample is affected by both mechanisms (Brownian- and collision-induced yielding) resulting in a double peak of $G_{1}^{\prime \prime}$ and two minima of higher harmonics, a feature that is detected for the first time in simple hard sphere glasses.

The rich mechanical response of a model hard sphere glass revealed in LAOS as a function of frequency, bridging the Brownian and non-Brownian regimes, may provide insights for the understanding of systems with more complicated interparticle interactions. These include both soft particle glasses that have been currently attracting significant interest (see, for example, Ref. [19]) as well as other pastes, slurries, particle gels, jammed emulsions, and metallic glasses. 
We thank A. B. Schofield for the particles and acknowledge funding from EU FP7-Infrastructures 'ESMI' (CP\&CSA-2010-262348) and Greek "Thales" Project "COVISCO".

*Corresponding author. georgp@iesl.forth.gr

[1] J. Mewis and N.J. Wagner, Colloidal Suspension Rheology, Cambridge Series in Chemical Engineering (Cambridge University Press, New York, 2012).

[2] R. G. Larson, The Structure and Rheology of Complex Fluids (Oxford University Press, Oxford, 1999).

[3] P. Pusey and W. van Megen, Nature (London) 320, 340 (1986).

[4] P. Pusey, in Liquids, Freezing and Glass Transition: Proceedings Les Houches Summer School, 1989, edited by J. P. Hansen, D. Levesque, and J. Zinn-Justin (Elsevier, Amsterdam, 1991).

[5] G. Petekidis, D. Vlassopoulos, and P. N. Pusey, J. Phys. Condens. Matter 16, S3955 (2004).

[6] R. Besseling, E. R. Weeks, A. B. Schofield, and W. C. K. Poon, Phys. Rev. Lett. 99, 028301 (2007).

[7] N. Koumakis, M. Laurati, S. U. Egelhaaf, J. F. Brady, and G. Petekidis, Phys. Rev. Lett. 108, 098303 (2012).

[8] G. Petekidis, A. Moussaid, and P. N. Pusey, Phys. Rev. E 66, 051402 (2002).

[9] G. Petekidis, D. Vlassopoulos, and P. Pusey, Faraday Discuss. Chem. Soc. 123, 287 (2003).

[10] K. Hyun, M. Wilhelm, C. O. Klein, K. S. Cho, J. G. Nam, K. H. Ahn, S. J. Lee, R. H. Ewoldt, and G. H. McKinley, Prog. Polym. Sci. 36, 1697 (2011).
[11] T. G. Mason and D. A. Weitz, Phys. Rev. Lett. 75, 2770 (1995).

[12] J. M. Brader, M. Siebenbuerger, M. Ballauff, K. Reinheimer, M. Wilhelm, S. J. Frey, F. Weysser, and M. Fuchs, Phys. Rev. E 82, 061401 (2010).

[13] P. Sollich, Phys. Rev. E 58, 738 (1998).

[14] D. Pine, J. Gollub, J. Brady, and A. Leshansky, Nature (London) 438, 997 (2005).

[15] P. Ballesta, R. Besseling, L. Isa, G. Petekidis, and W. Poon, Phys. Rev. Lett. 101, 258301 (2008).

[16] R. Besseling, L. Isa, P. Ballesta, G. Petekidis, M. E. Cates, and W. C. K. Poon, Phys. Rev. Lett. 105, 268301 (2010).

[17] C. Lopez-Barron, L. Porcar, A. Eberle, and N. Wagner, Phys. Rev. Lett. 108, 258301 (2012).

[18] M. P. Lettinga, P. Holmqvist, P. Ballesta, S. Rogers, D. Kleshchanok, and B. Struth, Phys. Rev. Lett. 109, 246001 (2012).

[19] N. Koumakis, A. Pamvouxoglou, A. S. Poulos, and G. Petekidis, Soft Matter 8, 4271 (2012).

[20] See Supplemental Material at http://link.aps.org/ supplemental/10.1103/PhysRevLett.110.178301 for description of BD simulations and movie of stresses and structures within LAOS.

[21] D. R. Foss and J. F. Brady, J. Rheol. 44, 629 (2000).

[22] K. N. Pham, G. Petekidis, D. Vlassopoulos, S. U. Egelhaaf, W. C. K. Poon, and P. N. Pusey, J. Rheol. 52, 649 (2008).

[23] N. Koumakis and G. Petekidis, Soft Matter 7, 2456 (2011).

[24] K. Miyazaki, H.M. Wyss, D. A. Weitz, and D. R. Reichman, Europhys. Lett. 75, 915 (2006).

[25] M. Fuchs and M. E. Cates, J. Rheol. 53, 957 (2009).

[26] E. Saltzman, K. Yatsenko, and G. Schweizer, J. Phys. Condens. Matter 20, 244129 (2008).

[27] A. Sierou and J. F. Brady, J. Fluid Mech. 506, 285 (2004). 